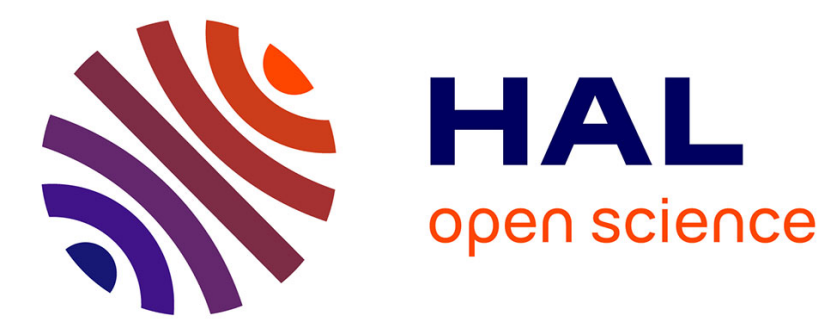

\title{
Airplane tire inspection by image processing techniques
} Igor Jovančević, Al Arafat, Jean-José Orteu, Thierry Sentenac

\section{To cite this version:}

Igor Jovančević, Al Arafat, Jean-José Orteu, Thierry Sentenac. Airplane tire inspection by image processing techniques. 5th Mediterranean Conference on Embedded Computing (MECO'2016), Jun 2016, Bar, Montenegro. 10.1109/MECO.2016.7525733 . hal-01351750

\section{HAL Id: hal-01351750 \\ https://hal.science/hal-01351750}

Submitted on 4 Aug 2016

HAL is a multi-disciplinary open access archive for the deposit and dissemination of scientific research documents, whether they are published or not. The documents may come from teaching and research institutions in France or abroad, or from public or private research centers.
L'archive ouverte pluridisciplinaire HAL, est destinée au dépôt et à la diffusion de documents scientifiques de niveau recherche, publiés ou non, émanant des établissements d'enseignement et de recherche français ou étrangers, des laboratoires publics ou privés. 


\title{
Airplane tire inspection by image processing techniques
}

\author{
Igor Jovančević, Al Arafat, Jean-José Orteu, Thierry Sentenac \\ ICA (Institut Clement Ader) \\ Universite de Toulouse; CNRS, INSA, UPS, Mines Albi, ISAE \\ Albi, France \\ Email: igor.jovancevic@mines-albi.fr
}

\begin{abstract}
In this paper, we propose a new approach to detect and inspect airplane tires. We use normalized correlation based template matching to detect tires and GrabCut segmentation method to extract them from the background. We inspect the tires condition by investigating the tire treads. Ridge based intensity profiling gives satisfying inspection results.
\end{abstract}

Keywords-image processing; inspection; PTZ camera; template matching; intensity profiling

\section{INTRODUCTION}

Airplanes are periodically inspected, either on the airport tarmac between flights or in a hangar during maintenance operations. These inspections are done by human operators, mainly visually and sometimes with some inspection tools. In order to make the inspection quicker, more exhaustive and more accurate, as well as for traceability reasons, a multipartners research project is being carried on to develop a mobile robot equipped with several sensors (cameras and 3D scanner) to perform an automatic inspection of an airplane. The chosen approach is based on an autonomous mobile robot on the ground, capable to communicate with human operators and infrastructures around the aircraft. In addition to the long range navigation task, at each checkpoint, the robot performs an inspection task of several items (tires, probes, doors, etc.) [1].

In a typical scenario, navigation algorithms lead the robot to a checking position which is localized in the aircraft model. Further, automated image acquisition is provided by controlling Pan-Tilt-Zoom (PTZ) camera and pointing it towards the item to be inspected. Inspection is performed by processing the images taken by the camera.

This paper describes an insight to construct an image processing pipeline to detect and inspect airplane tires as a mandatory requirement of the project. Existing image processing techniques are employed in order to isolate and examine the condition of airplane tires.

In the literature, to our knowledge, there is no many papers that concern the tire inspection relying on image processing. Xiang et al. [2] used Dictionary-based representation to detect tire defects. Chen et al. [3] inspected tire tread defects using image processing and pattern recognition methods.
This paper possesses two main contributions. The first contribution is to introduce ridge based inspection of tire treads. We use intensity profile of an image to inspect the treads condition.

The second contribution is in developing the entire image processing pipeline for detecting and inspecting tires robust to the noise in relative pose of the robot with respect to the airplane and its surrounding environment.

Our model uses Bilateral filter to smooth the original captured image and the template patch. It further employs normalized correlation based template matching to detect the tire region in the image. To isolate tire from the background we are running the GrabCut [4] segmentation method. To automatically generate foreground bounding box required for GrabCut algorithm, we rely on the template matching output. By utilizing the rough estimation of the robot pose, and the known position of the camera on the robot, we are able to project the known simplified tire model on the image. The approximate size of the tire is therefore available and used in this phase to help estimate the bounding box size in the image.

After segmenting the tires, our model removes small contours and performs morphological operations to achieve neat segmentation result. At the end, it inspects the segmented images by differentiating out the tire treads using ridge detection and shows the position of the worn tread on the tire by using intensity profiling.

The steps of the method are given in section II. Section III shows our experimental results. Finally, we draw conclusions in Section IV.

\section{DEVELOPED FRAMEWORK}

We assume that both tires are visible in the image (Fig. 1),

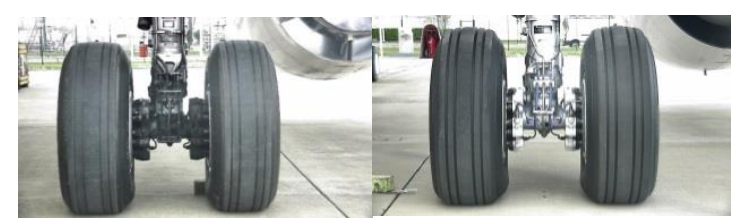

Figure 1. Captured images using PTZ camera $\begin{array}{ll}\text { a) Tires in bad condition } & \text { b) Tires in good condition }\end{array}$ 
which is usually the case, despite of the noisy localization result.

Our method consists of three main steps: detection, segmentation and inspection. The flowchart in Fig. 2 shows the steps.

\section{A. Detection}

In the captured images, although the tires seem to be two separate dark regions, the detection becomes challenging due to the non-homogenous intensities. Tire color varies significantly depending on the tire age as well as on the amount of dust present on the tire. Moreover, shadows, varying

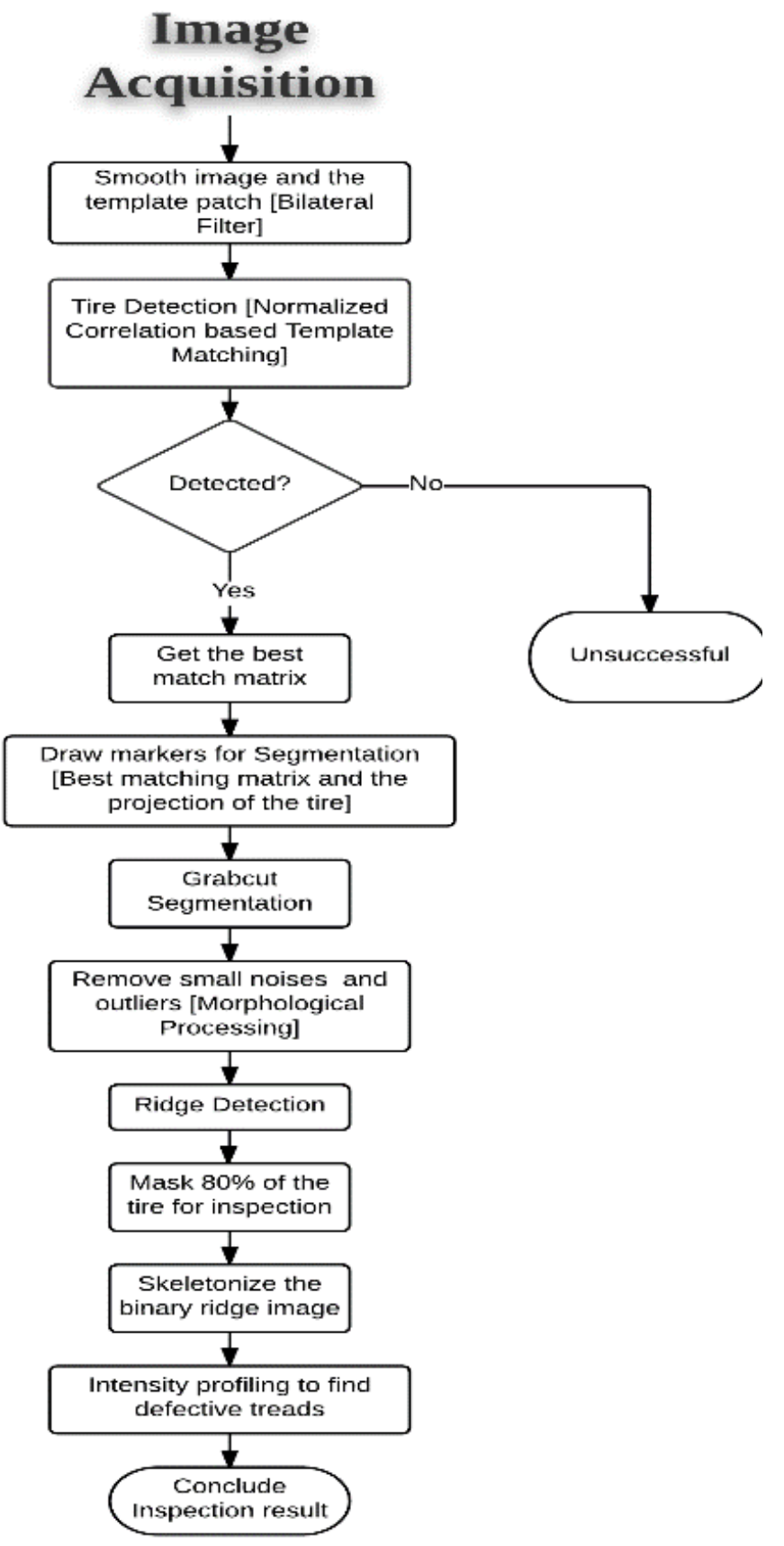

Figure 2. Work flow pipeline. illumination caused by weather conditions, and altering surrounding environment (tarmac, hangar) add more variability. In detection and segmentation phases, high precision is required because the inspection is done on the segmented regions.

We use normalized cross correlation [Equation (1)] based template matching to detect the patch of a tire:

$$
R(x, y)=\frac{\sum_{x^{\prime}, y^{\prime}}\left(T\left(x^{\prime}, y^{\prime}\right) * I\left(x+x^{\prime}, y+y^{\prime}\right)\right)}{\sqrt{\sum_{x^{\prime}, y^{\prime}} T\left(x^{\prime}, y^{\prime}\right)^{2} * \sum_{x^{\prime}, y^{\prime}} I\left(x+x^{\prime}, y+y^{\prime}\right)^{2}}} .
$$

Before that, we remove the non-uniformity by using Bilateral filter. Bilateral filter smooths the original image and the template image but preserves the edges. It can be seen in Fig. 3 that the high response is obtained in the tires region.

The pattern of tire treads does not vary for a specific airplane model. It also does not have sharp details on the tire surface. Moreover, we do not have examples with very rotated tires. Thus, we use Template matching exhaustive search to detect the tire patch. Additionally, normalized cross-correlation similarity metric is invariant to global brightness changes.

We also evaluated alternative approach relying on Saliency based object locator to detect the middle part of the landing gear (between the two tires). Fig. 4 shows an example. We were inspired to use saliency estimation based on region covariances [5]. We have the accuracy of $82.20 \%$ in detecting the middle part of the landing gear using saliency estimation and picking the most salient region. Still, template matching

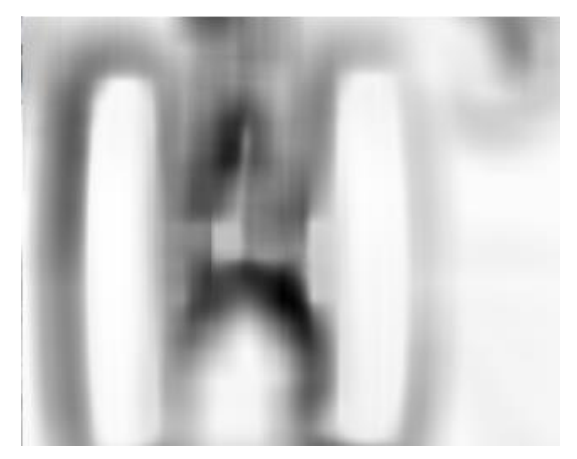

Figure 3. Similarity score image

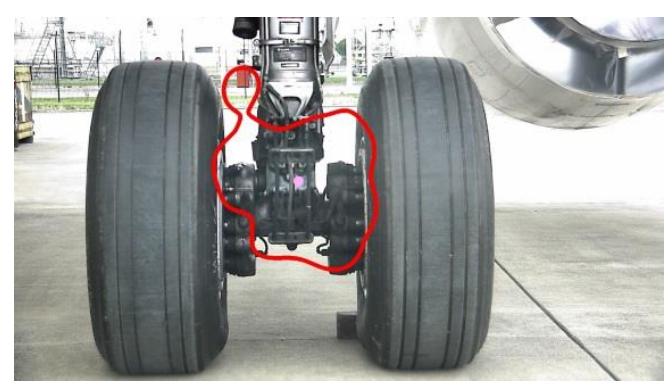

Figure 4. Saliency based detection of the middle part of the landing gear. 
has overperformed saliency based approach.

\section{B. Segmentation}

This section describes the segmentation of the two tires regions from the background. Inspection of the tires requires the accurately segmented tires regions. The tire-alike shadow regions and the presence of other dark objects around the tires complicate the task of segmenting the tires.

Color based Region growing segmentation method suffers from over-segmentation since it is limited to local homogeneity criteria without having the global perspective (Fig. 5a).

We also experience local irregularities in the gradient which causes the interactive image segmentation method, for our case, Marker based Watershed, to produce over-segmented results (Fig. 5b). As markers, we use two regions indicated in Fig. 6.

To suite our requirement, we use GrabCut segmentation method to segment the tires from the background. Nevertheless, GrabCut algorithm requires bounding box around the object to be segmented. By binarizing the similarity score image shown in Fig. 3, we obtain regions likely belonging to tires (Fig. 6). The region most likely belonging to a tire is obtained as a best match (Fig. 6 left ellipse). The second tire region is identified as the one with similar orientation to the first tire (Fig. 6 right ellipse). By isolating these two regions, we obtain the bounding boxes around both tires for GrabCut algorithm.

After extracting the tires from the background, we remove the small disjoint outliers using morphological opening and closing.

GrabCut segmentation method successfully combines both the texture and the contrast information. It estimates the color
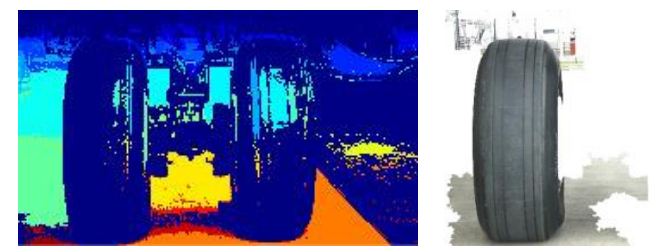

Figure 5. Alternative approaches

a) Region growing

b) Watershed
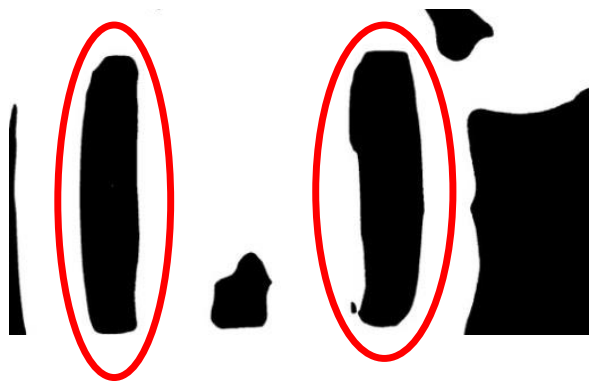

Figure 6. Binarizing similarity score image distribution of the foreground and background using Gaussian mixture model. It is Markov random field based segmentation method, meaning it favorizes neighboring pixels to have the same labels. End result is shown in Fig. 7.

\section{Inspection}

This section illustrates the decision making step. Outcome should show the condition of the tires, whether they are worn out or not. The decision is carried out by examining the health (depth) of the treads of each tire. If the treads fade, then the method informs the operator that the tire should be changed. We use ridge based intensity profiling of the tires to inspect the treads health.

Segmented tires have closely homogeneous regions, except the treads. Fig. 8 shows gradient magnitude of a tire surface. We observe changes of intensities along the treads. Edge detection detects the significant intensity changes, the steepness of slope at each point. Thus, for our instances, edge detection results in having two fringes along each tread, since we get changes of intensity values on the treads twice (from high to low and again from low to high). Fig. 8 shows why edge detection does not suit our problem definition.

Ridges (intensity valleys), on the contrary, are useful to distinct out the treads from the tire (Fig. 9a).

Firstly, we detect gradient changes (Fig. 8b) and then with dilation we are merging double parallel edges, to get the ridges (Fig. 9a).

We suppress border ridges by morphological operations. Further, we thin the ridges and extract skeletons from them. Obtained skeletons of treads are shown in Fig. 9b. We prune

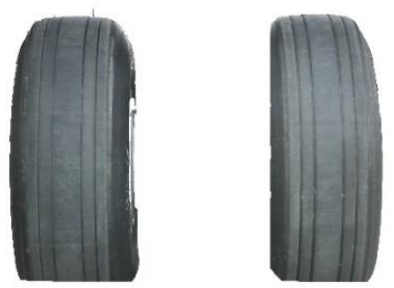

Figure 7. Segmented tires using GrabCut.

the skeleton image to remove the small branches and noise.

On the resultant skeleton image, we use intensity profiling
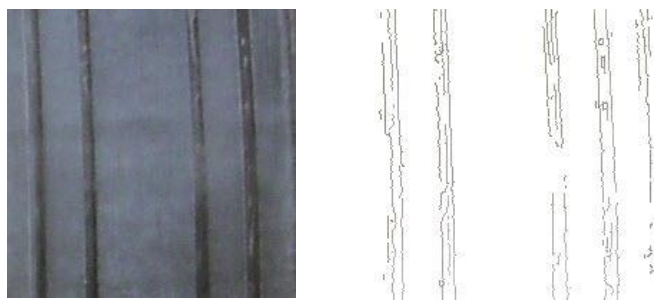

Figure 8. Edge detection to detect tire treads.
a) Treads
b) Edges 
to investigate the condition of the treads. With the profile line (red line in Fig. 9b) we are scanning the skeleton image from top to bottom of the tire region. We receive a set of onedimensional signals with peaks at the presence of treads. If the known pattern of treads is not found in the profile, the system reports a discontinuity of treads. The decision about changing the tire depends on the size of the discontinuity.

\section{RESULTS}

Fig. 10 shows our inspection result where the blue stars are indicating discontinuities in treads on the left or right from the stars.

The approach is demonstrated on three variants of Airbus A320 model, i.e. 12 tires. The images were taken in hangar as well as outside on the tarmac with different weather conditions.

The accuracy for our detection is $97.3 \%$ on our dataset of 118 images. Inspection approach has shown false negative rate (FNR) of $0 \%$ with acceptable false positive rate (FPR) of $8 \%$. Inspection dataset contains 52 worn out tires and 58 tires in good condition. We experience problems in the case of strong backlight, notably when the robot is placed under the fuselage.

\section{CONCLUSIONS}

We present an original approach to detect and inspect airplane tires based on existing image processing techniques. Our experiment shows the tire region detection accuracy of

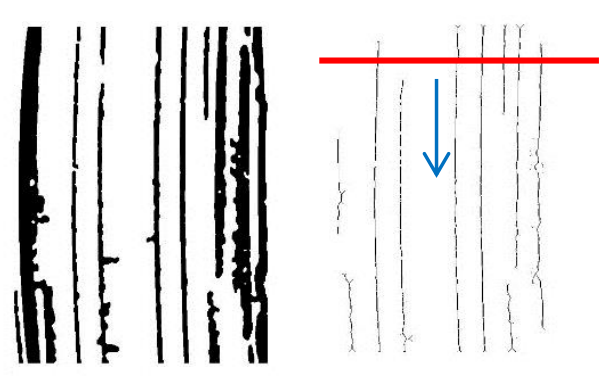

Figure 9.

(a) Ridges on the tire

(b) Skeleton of ridges
96.61\% and inspection accuracy with $8 \%$ FPR and $0 \%$ FNR. Our proposed pipeline combines template matching and GrabCut segmentation which in most of the cases, is robust in partitioning out the tires only. Our inspection model, however, can inspect the tire treads along the frontal tire surface only and is not yet robust to inspect the treads on the very top and very bottom part of the tire when the treads become curved.

Our method shows a sequence of operations to detect and inspect tires which has significant influence in airplane industry. We are currently working on increasing the dataset and evaluating binary classification techniques.

\section{ACKNOWLEDGEMENT}

This work is part of the AIR-COBOT project (http://aircobot.akka.eu) approved by the Aerospace Valley world competitiveness cluster. The authors would like to thank the French Government for the financial support via the Single Inter-Ministry Fund (FUI). The partners of the AIR-COBOT project (AKKA TECHNOLOGIES, Airbus Group, ARMINES, 2MoRO Solutions, M3 SYSTEMS and STERELA) are also acknowledged for their support.

\section{REFERENCES}

[1] I.Jovančević, S.Larnier, J-J. Orteu, T. Sentenac, “Automated exterior inspection of an aircraft with a pan-tilt-zoom camera mounted on a mobile robot" Journal of Electronic Imaging vol. 24(6), 061110, Nov. 2015.

[2] Y. Xiang, C. Zhang, and Q. Guo, "A Dictionary-based Method for Tire Defect Detection", Proc. IEEE Intl. Conf. on Information and Automation, Hailar, China, pp. 519 - 523, Jul. 2014.

[3] P. Chen, G. D. Shubinsky, K-H. Jan, C-A. Chen, O. Sidla, W. Poelzleitner, "Inspection of tire tread defects using image processing and pattern recognition techniques.” Proc. SPIE 2063, Vision, Sensors, and Control for Automated Manufacturing Systems, Nov. 1993.

[4] C. Rother, V. Kolmogorov, A. Blake, "GrabCut: interactive foreground extraction using iterated graph cuts", ACM Transactions on Graphics, Proc. ACM SIGGRAPH, vol. 23(3), pp. 309-314, Aug., 2004.

[5] E. Erdem, A. Erdem, "Visual saliency estimation by nonlinearly integrating features using region covariances.," Journal of Vision, vol.13(4), pp. 11-11, Jan. 2013.

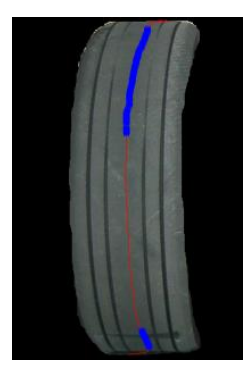

Figure 10. Inspection of tire treads. 